\title{
Optimisation of Cryo-SEM and how to Minimize Artefacts
}

\author{
M. A. Carey
}

Gatan UK, 25 Nuffield Way Abingdon Oxon, OX14 1RL, UK

Cryo-SEM is a popular technique for imaging a variety of hydrated samples, for example, tissues, plants, food stuffs, soft solids, liquids etc. One important aspect for a cryomiscoscopist is to recognise and interpret artefacts that can be associated with the technique. The artefacts that are associated with cryo-SEM can occur at each stage of the preparation process, that is, cryo-fixation, fracturing, sublimation and coating. In addition, surface contamination can also occur. Each area for potential artefacts will be covered and how to minimize their occurrence discussed.

Samples for cryo-SEM are usually plunged into liquid nitrogen slush to cryo-fix them. Whilst this procedure is fine for the surface analysis of samples and those of low water content, it is inadequate for high water content samples, especially liquids and those that require fracturing, as large ice crystals invariably pervade the structure. Damage caused by the formation of ice crystals present very characteristic features. To minimize ice crystal damage to a sample improvement in the cooling rate must be employed. Improvements to methodology will be discussed and a cost effective alternative to high pressure freezing will be shown.

Fracturing is a very simple process and easily performed using a $\mathrm{LN}_{2}$ cooled scalpel blade. Fracturing a frozen sample is a valuable technique to reveal internal microstructure and so provide further information about a sample. The artefact that is most commonly encountered during fracturing is scrape marks across the sample surface. A simple method to over come this problem will be provided.

Sublimation is a process where water is removed from the surface of a sample ( either the true surface and that revealed by fracturing) to enhance detail. Sublimation rate i.e. speed of removal of surface water is dependant on several factors: type of specimen and its water content (whether bound or free), temperature, time and vacuum. Of these variables temperature and time are the easiest for the microscopist to manipulate. Too high a temperature can result in collapse of structure whilst too low can provide data devoid of fine detail. Examples of both will be shown with suggestions on how to optimise the process.

Surface contamination can occur: before the sample is cryo-fixed, during sample manipulation after fixation, during transfer and during imaging in the electron microscope. Topics for discussion will include: improvements to sample handling, use of a high performance transfer device and the importance of effective de-contaminators. 